\title{
Référentiel métier-compétences ou comment structurer le métier d'urgentiste
}

\author{
Trade-skills referential: how to organize the emergency doctor's trade
}

\author{
J. Schmidt \\ C SFMU et Springer-Verlag France 2012
}

À l'aune de la transformation de l'essai marquant le passage du diplôme d'études spécialisées complémentaires (DESC) de médecine d'urgence vers un DES de médecine d'urgence, il fallait réécrire les fondamentaux.

Si j'ose ce langage rugbystique, loin de toute activité scientifique a priori, il me parait possible de comparer ces deux activités : beaucoup d'engagement sur le terrain avec $\mathrm{du}$ « beau jeu » quand rien n'est laissé au hasard et énormément de travail préparatoire en amont. La rédaction de deux référentiels métier-compétences dans le présent numéro des Annales françaises de médecine d'urgence témoigne des réflexions et travaux d'amont qui accompagnent la construction de notre métier.

Le référentiel pour la spécialité de médecine d'urgence précise pour les plus jeunes les ambitions de notre spécialité encore naissante et souligne pour les plus anciens le chemin parcouru au fil des années [1]. Ce référentiel nous rappelle utilement le champ de l'exercice de la médecine d'urgence avant de détailler les compétences et les aptitudes comportementales requises de l'urgentiste. Il place ce dernier au cœur du métier décliné sur la régulation médicale, sur les soins d'urgence sur place et pendant le transport, et sur la prise en charge des patients dans les services d'urgences et les unités d'hospitalisation de courte durée. Ce référentiel contribuera indéniablement à harmoniser les objectifs de stage des services validant le parcours des internes se destinant à la médecine d'urgence. Enfin, notre référentiel est conforme aux attentes de l'European Society for Emergency Medicine (EuSEM) et souligne de fait l'intégration souhaitée de la médecine d'urgence française dans une dimension européenne où elle devra défendre ses spécificités et ses valeurs.
Le référentiel pour la surspécialité de médecine d'urgence pédiatrique proposée par nos amis pédiatres rappelle, s'il le fallait, la réalité de terrain [2]. Nous n'ignorons pas que, hormis quelques exceptions propres aux hôpitaux de grande taille disposant de structures spécifiques d'urgences pédiatriques, l'accueil pédiatrique se fait dans la très grande majorité dans nos services d'urgences au sein de locaux partagés avec l'accueil des adultes. Si le référentiel pour la spécialité de médecine d'urgence prévoit une formation de qualité pour l'urgentiste issu du DES, il apparait cohérent d'aboutir à la création d'un DESC de type I de médecine d'urgence pédiatrique. Ce DESC complèterait tant la formation des pédiatres à la médecine d'urgence qu'il enrichirait celle des urgentistes à la pédiatrie ; la bonne recette en somme pour améliorer les connaissances et les compétences des urgentistes, et par là-même la qualité de soin que nous devons aux nourrissons et aux enfants.

Je tiens à remercier vivement l'ensemble des auteurs et des partenaires de la médecine d'urgence ayant contribué à la rédaction de ces deux référentiels qui consolident les fondations de la construction de notre métier.

Je vous invite maintenant à prendre place à l'ouverture...

\section{Références}

1. Nemitz B, Carli P, Carpentier F, Ducassé JL, et al (2012) Référentiel métier-compétences pour la spécialité de médecine d'urgence. Ann Fr Med Urg 2:125-38

2. Mercier JC, Martinot A, Parez N, Titomanolio L, et al (2012) Référentiel métier-compétences pour la surspécialité de médecine d'urgence pédiatrique. Ann Fr Med Urg 2:139-46

\author{
J. Schmidt $(\bowtie)$ \\ Pôle Samu-Smur-Urgences, CHU de Clermont-Ferrand, 58, rue \\ Montalembert, F-63003 Clermont-Ferrand Cedex 01, France \\ e-mail : jschmidt@chu-clermontferrand.fr
}

Faculté de médecine, université d'Auvergne Clermont, 28, place

Henri-Dunan, F-6300 BP 38 Clermont-Ferrand cedex 1, France 